\title{
CONSIDERAÇÕES ACERCA DOS ESTADOS REACIONAIS DO PORTADOR DE HANSENÍASE NO MUNICÍPIO DE ITAJAÍ
}

\author{
Jucelene Marchi Blatt
}

\begin{abstract}
RESUMO: A presente pesquisa teve por objetivo, compreender e avaliar os estados reacionais de pacientes portadores de hanseníase e o tratamento das reações. O método de abordagem do estudo foi indutivo, tendo como base o referencial bibliográfico, tomou-se como fonte de dados os prontuários dos portadores de hanseníase, disponíveis no programa de controle de hanseníase da Secretaria Municipal de Saúde de Itajaí. Através deste trabalho com os 78 pacientes inscritos no programa sujeitos da pesquisa, observou-se que: $60,3 \%$ tem de $15-49$ anos; $65,4 \%$ são do sexo masculino; 70,5\% manifestam a forma Virchowiana da doença; $60,3 \%$ apresentam estados reacionais durante tratamento; metade destes aproximadamente apresentaram quadro clínico de neurite, tendo sido tratados com prednisona, também se observou outros sintomas clínicos em escala menor como o eritema nodoso hansênico, e o tratamento de escolha sempre que possível é a talidomida; e 11,5\% apresentaram para efeitos ao tratamento com multidrogas. A avaliação dos estados reacionais indica que, mais da metade dos portadores em tratamento apresentam esta manifestação imunológica. É julgada pela literatura como conseqüência da doença e possível reação ao esquema poliquimioterápico.
\end{abstract}

PALAVRAS-CHAVE: hanseníase, estados reacionais, talidomida 


\section{INTRODUÇÃO}

A pesquisa sobre as manifestações imunológicas dos portadores de hanseníase e o tratamento destes estados reacionais no programa de hanseníase da região de Itajaí (SC)que compreende a Associação dos Municípios da Região da Foz do Rio Itajaí - Baln. Camboriú, Bombinhas, Camboriú, Itajaí, Itapema, Luiz Alves, Navegantes, Penha, Piçarras, Porto Belo, justificou-se por razões práticas e teóricas.

Na prática por trabalhar no laboratório microregional de Itajaí, que é o único de referência na região da AMFRI, com garantia para auxílio diagnóstico da hanseníase e outras doenças.

Teoricamente a preocupação em fazer a presente pesquisa foi pelo limitado número de estudos publicados sobre os estados reacionais dos portadores de hanseníase em tratamento com multidrogas (PQT: RFM - Rifampicina, CFZ - Clofazimina, DDS - Dapsona). No caso do programa de hanseníase de Itajaí e região foi possível reunir dados descritivos, para fins de análise, do conjunto de pacientes a partir dos prontuários.

A resposta imune dos portadores desta infecção hansênica são inicialmente do tipo celular (linfócito T) e segundo do tipo humoral (linfócitos B). Alguns estudos tem mostrado este papel imunológico relevante, visto que, nesta doença, ambos os aspectos estão intensamente presentes.

A reação imune celular após a infecção com Mycobacterium leprae é complexa, resulta em ativação do macrófago que são considerados responsáveis por induzir um aumento na habilidade de limitar a multiplicação bacteriana ou por matar o microorganismo intruso e assim essencial para imunidade protetora e resistência contra à infecção. Todavia a reação imune celular é também altamente complexa, envolvendo a geração de vários subgrupos de linfócitos T com várias funções. Algumas destas induz resistência, visto que um recrutamento muito intenso de células supressoras $T$ podem inibir o desenvolvimento da imunidade e podem assim ser um importante elemento no desenvolvimento de formas multibacilares da doença (HARBOE, 1985).

O sistema de defesa contra o Mycobacterium leprae está baseado na presença de uma imunidade celular, estes são os linfócitos timo-dependentes (T), que são sensibilizados pelo antígeno e depois ativam os macrófagos, este sistema parece ser falho na hanseníase Virchowiana. (COMITÉ EXÉCUTIF INTERNATIONAL DE L'ORDRE S.M. DE MALTE À GENĖVE, 1988). 
A evolução crônica da hanseníase pode cursar às vezes com fenômenos agudos que são chamados episódios reacionais, que tem relação com o sistema imunológico do indivíduo. Esses episódios reacionais são dos tipos I e II MINISTÉRIO DA SAÚDE (1994, p. 39).

Na visão de (YODER, 1987), a reação reversa é uma reação de imunidade celular, há uma mudança no estado imunológico do paciente, levando a uma reação inflamatória com edema nas áreas da pele e nervos afetados pela doença.

Para HARBOE (1985); BRITTON (1992), há um desequilíbrio transitório no mecanismo imunoregulatório em pacientes com eritema nodoso hansênico, a relação entre células auxiliares positiva T4 e células supressoras T8 positivas em sangue periférico, aumenta o quadro clínico da doença e retorna ao normal quando as lesões cessam. O decréscimo nas células supressoras $\mathrm{T}$, pode induzir uma mudança na quantidade e/ou afinidade de anticorpos anti Mycobacteirum leprae, favorecendo a formação de complexos imunes. A liberação pelos macrófagos dos antígenos é responsável pela formação e deposição de imune complexos, que induz ao dano no tecido vivo e sintomas clínicos típicos como nódulos intracutâneos elevados e dolorosos, sintomas constitucionais e febre alta.

Na perspectiva de HARBOE (1985) os fatores genéticos do indivíduo são importantes na determinação de susceptibilidade e o tipo de resposta após a infecção com Mycobacteirum leprae e assim para o desenvolvimento e tipo da doença clínica.

\section{MATERIAL E MÉTODO}

\section{Método de abordagem}

O método de abordagem foi indutivo (LAKATOS e MARCONI, 1994), por reunir e aproximar dados numa avaliação geral sobre os efeitos do tratamento na hanseníase.

\section{Método de procedimento}

O método de procedimento foi monográfico (ibidem) por estudar um determinado grupo numa condição específica - a hanseníase, com a finalidade da compreensão dos estados reacionais nos portadores. 


\section{Técnica de coleta de dados}

Após a análise dos prontuários dos portadores dos diferentes tipos de hanseníase, registrou-se em ficha individual, dados referentes ao sexo, idade, formas da doença, e estados reacionais, para serem analisados como métodos estatísticos, analíticos.

\section{Fonte dos dados}

A fonte dos dados documentais foram os prontuários dos portadores de hanseníase inscritos no Programa de Controle de Hanseníase da Secretaria Municipal de Saúde de Itajaí. A sede do programa é à rua José Bonifácio Malburg, nº. 195, situado no PAM (Posto de Atendimento Médico), do Ambulatório Central da Prefeitura Municipal de Itajaí.

\section{RESULTADOS}

\section{Situação dos pacientes do programa de hanseníase de Itajaí e região}

A situação dos setenta e oito (78) pacientes do programa de hanseníase de Itajaí e região é descrita na (tabela 1 ) através dos indicadores, faixa etária, segudo forma clínica, (tabela 2) sexo, segundo as formas clínicas, estados reacionais (tabela 3).

Os dados foram colhidos em dezembro de 1995, pela própria pesquisadora, no arquivo do Programa de Hanseníase de Itajaí.

Tabela 1. Distribuição da freqüência dos portadores inscritos no programa de hanseníase por faixa etária segundo as formas clínicas. - Itajaí (SC)dez. 1995.

\begin{tabular}{lcccccc}
\hline \multirow{2}{*}{ Faixa } & \multicolumn{4}{c}{ Formas Clínicas } & \multirow{2}{*}{ Total } & \multirow{2}{*}{ Frequência } \\
\cline { 2 - 5 } Etária & Indeterminada & Tuberculóide & Dimorfa & Virchowiana & \\
\hline $5-14$ & - & - & - & 2 & 2 & $2,6 \%$ \\
$15-49$ & 2 & 5 & 12 & 28 & 47 & $60,3 \%$ \\
$50+$ & - & 1 & 3 & 25 & 29 & $37,2 \%$ \\
\hline Total & $\mathbf{2}$ & $\mathbf{6}$ & $\mathbf{1 5}$ & $\mathbf{5 5}$ & $\mathbf{7 8}$ & $\mathbf{1 0 0} \%$ \\
\hline
\end{tabular}

Fonte: PCH-SMSI 
Saúde e Sociedade 10(1):55-64, 2001

Tabela 2. Distribuição da freqüência dos portadores inscritos no programa de hanseníase por sexo segundo as formas clínicas. - Itajaí (SC) dez. 1995.

\begin{tabular}{|c|c|c|c|c|c|c|c|c|}
\hline \multirow{2}{*}{ Sexo } & \multicolumn{4}{|c|}{ Formas Clínicas } & \multicolumn{2}{|c|}{ Freq. } & \multirow[t]{2}{*}{ Total } & \multirow[t]{2}{*}{ Freq } \\
\hline & Indeterminada & Tuberculóide & Dimorfa & Virchowiana & M & $\mathbf{F}$ & & \\
\hline Masc. & - & - & - & - & - & - & & \\
\hline Fem. & 2 & - & - & - & - & 2,6 & 2 & 2,6 \\
\hline Masc. & - & 2 & - & - & 2,6 & - & & \\
\hline Fem. & - & 4 & - & - & - & 5,1 & 6 & 7,7 \\
\hline Masc. & - & - & 11 & - & 14,1 & - & & \\
\hline Fem. & & - & 4 & - & & 5,1 & 15 & 19,2 \\
\hline Masc. & - & - & - & 38 & 48,7 & - & & \\
\hline Fem. & - & - & - & 17 & - & 21,7 & 55 & 70,5 \\
\hline Total & - & - & - & - & 65,4 & 34,5 & 78 & 100,0 \\
\hline
\end{tabular}

Fonte: $\mathrm{PCH}-\mathrm{SMSI}$

Os dados acima nos levam a pensar que o diagnóstico da hanseníase geralmente é feito tardiamente, pelo elevado número de portadores Virchowianos, porque a forma inicial indeterminada e a dimorfa apresentam menor número de casos, seja por desinformação do paciente com relação aos sintomas clínicos, ou por erros de diagnósticos, mesmo sendo a forma $\vee$ multibacilar e com alto poder de transmissibilidade.

Tabela 3. Distribuição da freqüência de portador com presença de estados reacionais durante o tratamento com PQT, segundo a forma clínica. - Itajaí (SC), dez. 1995.

\begin{tabular}{l|l|l}
\hline \begin{tabular}{l|l} 
ESTADOS REACIONAIS SEGUNDO \\
FORMA CLÍNICA
\end{tabular} & $\begin{array}{l}\text { Número } \\
\text { de portadores }\end{array}$ & Frequência \\
\hline VIRCHOWIANA & 34 & 43.6 \\
DIMORFA & 8 & 10.3 \\
TUBERCULÓIDE & 5 & 6.4 \\
INDETERMINADA & - & - \\
AUSÊNCIA DE ESTADOS REACIONAIS & 31 & 39.7 \\
\hline TOTAL & $\mathbf{7 8}$ & $\mathbf{1 0 0 . 0}$ \\
\hline
\end{tabular}

Fonte: Prontuários 


\section{DISCUSSÃO SOBRE OS EFEITOS REACIONAIS NOS PACIENTES EM TRATAMENTO}

As manifestações clínicas relacionadas à doença, podem ser acompanhadas por estados reacionais, através do levantamento dos prontuários dos pacientes foi observado a presença de neurites e eritema nodoso durante tratamento o que pode caracterizar a presença destes surtos nos portadores.

As reações ocorrem normalmente nos primeiros seis meses de tratamento e quase sempre nos primeiros 12 meses MINISTÉRIO DA SAÚDE (1994).

Através do levantamento de dados obtidos da análise dos prontuários dos pacientes, observou-se que a forma Virchowiana é predominante com $70,5 \%$ dos casos. Entre os pacientes com sintomas reacionais da forma $\vee$ observou-se que $43,6 \%$ apresentaram estados reacionais durante o tratamento. Segundo dados da FIOCRUZ (SARNO et al), em 94 pacientes com hanseníase, (44\%) tiveram um ou mais episódios reacionais durante tratamento, sendo que $27,7 \%$ tiveram reações tipo II, no nosso levantamento obtivemos $43,6 \%$ com este tipo de reação.

As reações do tipo I, que ocorrem nos pacientes tuberculóides e tuberculóides dimorfos e indeterminados, tiveram incidência menor $7,7 \%$ de reações, segundo pesquisa da FIOCRUZ, 9,6\% dos pacientes apresentaram este tipo de reação. A forma dimorfa tuberculóide e tuberculóide também são acometidas por sintomas relacionados a estados reacionais, entre os portadores com presença de neurites observou-se que a forma dimorfa com incidência de $19,2 \%$ dos casos, apresentou $10,2 \%$ de sintomas relacionados a surtos reacionais.

Os sintomas clínicos apresentados em decorrência da manifestação dos estados reacionais são em $55,3 \%$ as neurites, seguidos pelo ENH e neurites nos portadores da forma $\vee(34,5 \%)$ e ENH com 10,6\%, sendo que dos 78 portadores apenas $39,7 \%$ não apresentaram estes episódios.

Em observação a levantamentos realizados em outros programas como da Ethiopia 1992, observou-se que 41,5\% dos portadores DV e V, apresentaram reações hansênicas, sendo que $36,6 \%$ tiveram reação reversa, 5\% eritema nodoso. Do pólo tuberculóide limítrofe, $21 \%$ tiveram reação reversa e nos tuberculóides nenhuma reação foi observada.

PANNIKAR, et al, (1990), relata 33,3\% de pacientes com reações, sendo que, 31\% ocorreram no primeiro ano de tratamento, obtendo 5\% eritema nodoso, 18\% reação reversa, 
Saúde e Sociedade 10(1):55-64, 2001

$10,3 \%$ com neurites e destes pacientes 11,3\% tiveram reação após alta por cura, e destes $77,8 \%$ ocorreram no primeiro ano após a alta. No levantamento realizado, dos 47 pacientes reacionais $53,3 \%$ tiveram neurite como sintoma clínico. Dos 78 portadores $33,3 \%$ apresentaram como único sintoma a neurite, sendo esta a responsável pelas incapacidades provocadas pela doença.

Os episódios reacionais estão relacionados basicamente com a reação imunológica do indivíduo a PQT, por isto praticamente a metade dos portadores apresentam este sintoma durante tratamento, reação esta que parece ser benéfica pela resposta do organismo aos antígenos presentes.

Segundo Fundação Osvaldo Cruz, (apud PDS/SC, sd.), 27, 3\% das reações hansênicas ocorrem após alta por cura, sendo que $80,4 \%$ ocorreram no primeiro ano de seguimento, o que comprova a relação entre uma grande carga bacilar quando em tratamento, e como esta limpeza bacilar é lenta, e se dá por conta do sistema fagocitário do indivíduo na ordem de 0,6 a 1,0 log/ano, a presença destes estados podem ocorrer após alta por cura. A presença contínua de antígenos de bacilos mortos constitui risco de reações reversas e eritema nodoso.

Os sintomas clínicos provenientes dos estados reacionais, também podem levar o portador a apresentar incapacidades físicas em decorrência das neurites, a pesquisa relata que $60,3 \%$ dos portadores foram acometidos por estes sintomas, observa-se nos levantamentos realizados por outros programas que, a presença de reações hansênicas em média é de 40\%. Entre as causas desta ocorrência está o alto índice baciloscópico encontrado nos doentes da nossa região, e sendo esta limpeza bacilar lenta, o índice de reações devem ser maiores em decorrência deste dado, pela presença de antígenos bacilares no organismo.

Quanto ao tratamento dos estados reacionais às drogas de escolha normalmente é a prednisona $(53,2 \%)$, para os portadores com neurites, seguida pela associação talidomida e prednisona em pacientes que além do ENH apresentam neurites $(34, \%)$ e talidomida para casos isolados de $\mathrm{ENH}(10,6 \%)$ dos portadores com hanseníase virchowiana.

Dados do $14^{\circ}$. CONGRESSO INTERNACIONAL DE HANSENÍASE (1993), indicam que 50 a $60 \%$ dos pacientes virchowianos(V), desenvolvem um ou mais episódios de eritema nodoso, mas em programas com detecção precoce somente 10 a 15\% dos pacientes apresentam este sintoma. Em nosso levantamento $10,6 \%$ dos portadores $V$ apresentaram eritema nodoso em tratamento. 


\section{CONCLUSÕES}

O estudo sobre as considerações acerca dos estados reacionais dos portadores de hanseníase, permite algumas ponderações significantes tais como:

- a incidência maior da doença em adultos jovens do sexo masculino, o que caracteriza em hipótese, a predisposição do homem a ter mais relações interpessoais, e uma maior exposição ao meio, e também pelo logo período de incubação da doença.

- as condições sócio-econômicas e o fator genético também parecem determinar a suceptibilidade à doença.

- a presença de estados reacionais durante o tratamento parece ser reflexo do comportamento imunológico do indivíduo, observou-se que $60,3 \%$ dos pacientes em tratamento apresentaram sintomas clínicos que caracterizam estes episódios. Entre as causas destes episódios parece ser o alto índice baciloscópico dos portadores.

- os sintomas relacionados a estes estados reacionais mais freqüentes são as neurites (55,3\%), manifestando-se em todas as formas da hanseníase, seguidas pelo eritema nodoso hansênico $(10,6 \%)$, também chamado de reação tipo II, encontrados apenas na forma multibacilar da doença.

- o medicamento de escolha para a reação tipo 1 é a prednisona, atuando como anti-inflamatório sistêmico e imunossupressor. Na reação tipo II, a talidomida é muito eficaz, e sempre que possível deve ser considerada a droga de primeira escolha pelo rápido efeito que exerce sobre o eritema nodoso hansênico. É um modulador da resposta imune do organismo e modificador da resposta inflamatória.

A principal implicação do estudo, é que resultou em um banco de dados, de algumas relações entre as variáveis do levantamento, que deverá ser continuado para subsidiar análises sobre a presença dos estados reacionais nos anos subseqüentes, após o término do tratamento com multidrogas.

O encaminhamento da proposta é, fornecer os resultados do presente trabalho à Secretaria Municipal de Saúde para novos estudos, e acima de tudo este trabalho constituiuse em um grande aprendizado acerca de como elaborar um trabalho científico, desvelando um pouco mais de uma doença tão estigmatizada, de alta incidência, com tantas repercussões sociais e que, apesar dos avanços da ciência continua a ser um dos graves problemas de saúde pública. 


\section{REFERÊNCIAS BIBLIOGRÁFICAS}

BRITTON, W. J. Immunology of leprosy. Trans. R. Soc. Trop. Med. Hig., 87:508-514, 1993.

COMITÉ EXÉCUTIF INTERNATIONAL DE L'ORDRE S.M. DE MALTE À GENÈVE. Immunologie de la lèpre. Acta Leprol., 6(2):34-7, 1988.

HARBOE, M. The immunology of leprosy. In: Hastings, R. C. et al. Leprosy. Churchill Livingstone, 1985. p.53-86.

LAKATOS, E. M.; MARCONI, M. de A. Fundamentos de metodologia científica. 3.ed. São Paulo: Atlas, 1994.

MINISTÉRIO DA SAÚDE. Guia de controle da hanseníase. 2.ed. Brasília: Fundação Nacional da Saúde, 1994.

PROGRAMA DE DERMATOLOGIA SANITÁRIA (PDS/SC). Frequência das reações hansênicas. Florianópolis, s.d. (documentário informativo).

PROGRAMA DE DERMATOLOGIA SANITÁRIA (PDS/SC). Situação epidemiológica e operacional da hanseníase em Santa Catarina. Florianópolis, 1995. (documentário informativo)

YODER, L. J. Manejo de reações em hanseníase. Rev. Star, (jan./fev.), 1987. 


\section{CONSIDERATIONS ON REACTIONAL STATES OF ITAJAI'S HANSEN'S CARRIERS}

SUMMARY: The present research had as objetive to undestand and to evaluate the reactional episodes of pacients who had hansen disease and the treatment of the reactions. The method of the approach of the study was made by indicement base the referential bibliografhy, it was taken as data the reports of pacients of hanse's avaible the control program of leprosy in the control of Itajaí. Through this work with the 78 pacients enrolled and subjects of this search it was observed that: $60,3 \%$ is between $15-49$ years of age; $65,4 \%$ presents reactional episodes during treatment, half of these approximatly presented clinic symptons of neuritis, have being treates with prednisona, it was also observed other smaller clinic symtoms as erythema nodosum leprosum and the first choice of treatment is always with if possible is a talidomida. The evaliation of the reactional episodes indicates that more than half of the pacients in treatment present this immunological manifestacion. It is judged by the literature as the disease consequence and a possible reaction to the polichemiotherapy.

KEY WORDS: leprosy, reactional episodes, talidomida 